\title{
The Significance of Impact Factor
}

\section{Joshi BR}

The impact factor was devised by Eugene Garfield, the founder of the Institute for Scientific Information. Impact factor of an academic journal is a measure reflecting the yearly average number of citations to recent articles published in that journal. Journals with higher impact factors are often deemed to be more important than those with lower ones.

In any given year, the impact factor of a journal is the number of citations, received in that year, of articles published in that journal during the two preceding years, and divided by the total number of articles published in that journal during the two preceding years. The calculation always uses two complete and known years of item counts.

The impact factor is used to compare different journals within a certain field. It cannot be used to compare journals across disciplines because citation counts have highly skewed distributions. Impact factors are sometimes used to evaluate not only the journals but the papers therein, thereby devaluing papers in certain subjects.

A journal can adopt editorial policies to increase its impact factor. Journals may publish a larger percentage of review articles which are cited more than research papers. Some journals may invite exclusively senior scientists to publish 'citable' papers to increase the journal impact factor. Journals may publish a large portion of papers, or at least the papers expected to be highly cited early in the calendar year to give more time to gather citations.

Coercive citation is a practice in which an editor forces an author to add extraneous citations to an article before the journal will agree to publish it, in order to inflate the journal's impact factor.

Impact factor is a journal metric and should not be used to assess individual researchers and institutions. It should be used cautiously for measuring and comparing the influence of entire journals. Citation distribution metrics is an alternative to impact factors. 